\title{
Non-boronized compared to boronized operation of ASDEX Upgrade with full-tungsten plasma facing components
}

\author{
A. Kallenbach, R. Dux, M. Mayer, R. Neu, T. Pütterich, V. Bobkov, J.C. Fuchs, \\ T. Eich, L. Giannone, O. Gruber, A. Herrmann, L.D. Horton, C.F. Maggi, H. Meis- \\ ter, H.W. Müller, V. Rohde, A. Sips, A. Stäbler, J. Stober and ASDEX Upgrade Team \\ Max-Planck-Institut für Plasmaphysik, EURATOM Association, Garching, GER- \\ MANY
}

\begin{abstract}
After completion of the tungsten coating of all plasma facing components, ASDEX Upgrade has been operated without boronization for 1 1/2 experimental campaigns. This has allowed the study of fuel retention under conditions of relatively low D co-deposition with low-Z impurities as well as the operational space of a full-tungsten device for the unfavourable condition of a relatively high intrinsic impurity level. Restrictions in operation were caused by central accumulation of tungsten in combination with density peaking, resulting in H-L backtransitions induced by too low separatrix power flux. Most important control parameters have been found to be the central heating power, as delivered predominantly by ECRH, and the ELM frequency, most easily controlled by gas puffing. Generally, ELMs exhibit a positive impact, with the effect of impurity flushing out of the pedestal region overbalancing the ELM induced W source. The restrictions of plasma operation in the unboronized $\mathrm{W}$ machine occured predominantly under low or medium power conditions. Under medium-high power conditions, stable operation with virtually no difference between boronized and unboronized discharges was achieved. Due to the reduced intrinsic radiation with boronization and the limited power handling capability of VPS coated divertor tiles $\left(\approx 10 \mathrm{MW} / \mathrm{m}^{2}\right)$, boronized operation at high heating powers was possible only with radiative cooling. To enable this, a previously developed feedback system using (thermo-)electric current measurements as approximate sensor for the divertor power flux was introduced into the standard AUG operation. To avoid the problems with reduced ELM frequency due to core plasma radiation, nitrogen was selected as radiating species since its radiative characteristic peaks at lower electron temperatures in comparison to $\mathrm{Ne}$ and Ar, favouring SOL and divertor radiative losses. Nitrogen seeding resulted not only in the desired divertor power load reduction, but also in improved energy confinement, as well as in smaller ELMs.

e-mail: Arne.Kallenbach@ipp.mpg.de
\end{abstract}

PACS numbers: 52.25.Fi, 52.40.Hf, 52.55.Rk 


\section{Introduction}

With the 2007 experimental campaign, the ASDEX Upgrade tokamak completed its conversion into a device with $100 \%$ tungsten plasma facing components (PFCs) [1]. As final elements, $200 \mu \mathrm{m}$ thick vaccuum plasma-sprayed (VPS) tungsten coatings were installed in the outer divertor strike point region. All other PFCs are coated with 3-4 $\mu$ m thick physical vapour deposited (PVD) coatings [2]. In order not to compromise the results obtained for plasma operation in a high-Z device, no boronization was performed before startup and during the first 1 1/2 experimental campaigns. Old boron layers had been mechanically removed during the preceding vent as much as possible. Operation with no carbon tiles facing directly the plasma and without boronization allowed the study of fuel retention with low levels of fuel co-deposition with $\mathrm{B}$ and $\mathrm{C}$. On the other hand, the relatively high impurity level in the unboronized machine lead to restrictions of the $\mathrm{H}-$ mode operational space, since low-medium Z impurities dominate the physical sputtering of tungsten. In particular, ICRF heated discharges suffered from enhanced radiative losses due to tungsten sputtered by impurities accelerated in sheathrectified electric fields at the antenna limiters [3]. With the first boronisation in the second campaign with full-W coating, the intrinsic radiation level dropped and the power load at the outer target increased. It turned out that the power flux then could exceed the limit of about $10 \mathrm{MW} / \mathrm{m}^{2}$ set by a few lower quality thick VPS coated tiles. Feedback-controlled injection of nitrogen has been introduced as standard operational scenario for discharges with more than about 8 MW heating power to limit the divertor target power flux to the technically acceptable level. Exceeding of this level would lead to a soft pulse stop triggered by the video observation system.

This paper is organised as follows: Post campaign surface analysis results relevant to fuel retention are presented in the following chapter. The intrinsic impurity composition with and without boronization obtained from spectroscopic measurements is shown in chapter 3. Chapter 4 discusses the H-mode operational space and its limitations by tungsten depending on wall conditioning status. The tungsten source distribution and individual contributions are briefly summarised in chapter 5. Chapter 6 describes the feedback system used to raise the radiation level to obtain divertor-compliant power fluxes as intrinsically present with unboronized operation. Finally, some conclusions are drawn and a short outlook on future work is given.

\section{Campaign integrated deposited layers and fuel retention}

Post-campaign analysis of C and B layers on PFC surfaces as well as D retention measurements allowed the characterisation of fuel retention over the $\mathrm{C} \rightarrow \mathrm{W}$ PFC transition [4] [5]. Figure 1 shows a large reduction of $\mathrm{C}$ deposition in the inner divertor after $\mathrm{W}$ coating of the outer limiters, identifying these limiters as a dominant $\mathrm{C}$ source. The corresponding $\mathrm{D}$ codeposition exhibited a less pronounced reduction as expected from the $\mathrm{C}$ reduction due to the remaining $\mathrm{D}$ codeposition with B. The latter almost vanished after cleaning of the PFCs and non-boronized all-W operation. For these conditions, D retention in the outer divertor due to deep diffusion and trapping in $\mathrm{W}$ became the dominant retention mechanism, albeit at a much lower absolute level compared to a low-Z PFC device (reduction of the total trapped D inventory by a factor 5-10). The long term D retention in the full-W device from surface analysis was found to be $0.4-0.6 \%$ of the gas input [4]. This low value is compatible to in situ gas balance measurements. For a well analysed high density H-mode pulse [6], about $10 \%$ of the gas input is remaining in the vessel directly when the plasma current ends. Subsequent outgassing in the following minutes leads to zero retention within the uncertainty of the gas balance measurement of $\pm 3 \%$. The in-situ gas balance measurements revealed ceasing fuel storage of $\mathrm{D}$ after an initial discharge phase with a dynamic wall retention corresponding to about 44 monolayers when the geometrical surface of the torus is taken as effective area [6]. The deuterium atoms are assumed to be dissolved in the tungsten close to the surface. 
Extrapolating to ITER or a steady-state device, diffusion and trapping is the favourable retention mechanism compared to co-deposition. The fluence scaling of the retained fuel is expected to have approximately a square root dependence [7] due to its diffusive nature compared to the linear relation for the co-deposition. An open issue is the effect of neutron induced traps on the diffusive behaviour and trapping of tritum in tungsten. The traps are expected to increase the storage capacity of the material, but may also serve as diffusive barriers [8].
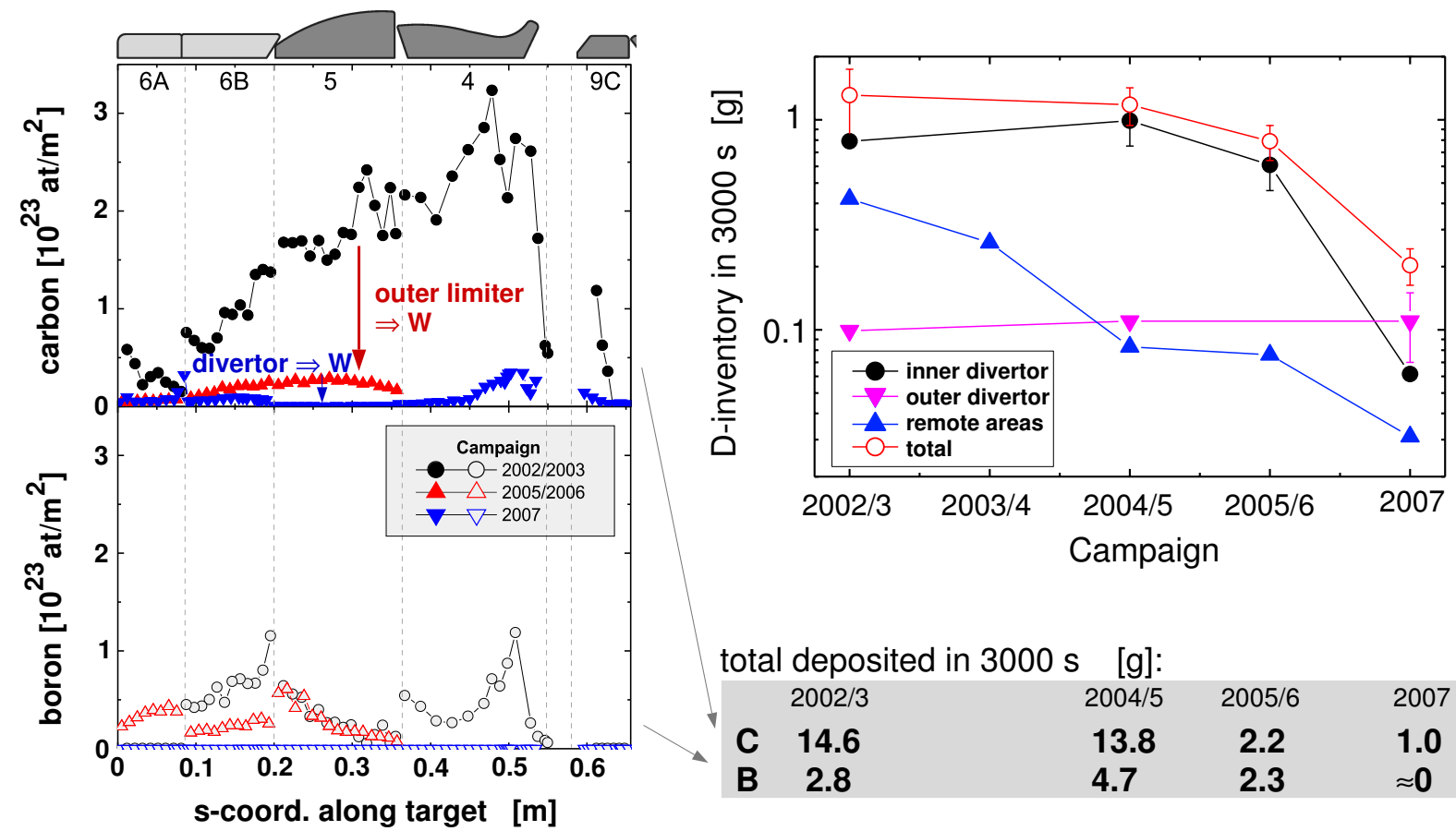

total deposited in $3000 \mathrm{~s} \quad[\mathrm{~g}]$ :

$\begin{array}{ccccc} & 2002 / 3 & 2004 / 5 & 2005 / 6 & 2007 \\ \text { C } 14.6 & 13.8 & 2.2 & 1.0 \\ \text { B } 2.8 & 4.7 & 2.3 & \approx 0\end{array}$

Figure 1. Deposited $C$ and B layer densities in the inner divertor during the transition from $C$ to $W$. In 2002/3, the inner heat shield and upper inner divertor were $W$ coated, the coating of the lower divertor before the 2007 campaign completed the transformation to full-W. Also shown is the temporal evolution of the D content for various plasma facing components. The table gives the total amount of deposited $C$ and $B$ in the inner divertor.

\section{Impact of boronization}

The impurity content pre- and post-boronization has been monitored by various spectroscopic systems. Figure 2 shows the temporal development of the most important intrinsic impurities $\mathrm{C}, \mathrm{O}$ and $\mathrm{F}$. Boron is hardly detectable before the boronization, CXRS measurements after boronization result in a typical B concentration at the pedestal top of $0.8 \%$, which slowly decays to $0.4 \%$ over 200 discharges. Despite considerable scatter in the impurity concentrations, the reduction of the $\mathrm{O}$ and $\mathrm{C}$ levels by the boronization is clearly visible. $\mathrm{C}$ experiences a strong reduction, but returns to about half the pre-boronization level after about 30-40 discharges. The general reduction of the $\mathrm{C}$ content was in fact less than originally expected for the full-W coating. Remaining sources, with unknown relative contributions, are arc traces cutting through the $4 \mu \mathrm{m} \mathrm{W}$ coating at the inner divertor baffle, photo-desorption from remote surfaces and the release of carbon from uncoated tile sides. Residual gas analysis suggests also the importance of erosion of $\mathrm{C}$ by oxygen due to $\mathrm{CO}$ and $\mathrm{CO}_{2}$ formation in the unboronised device. Erosion of carbon by oxygen could also partly explain the decrease of the $\mathrm{C}$ concentration after boronization: Oxygen is gettered by boron, thus reducing chemical erosion by formation of $\mathrm{CO}$ and $\mathrm{CO}_{2}$. Another contributing effect is the coating of carbon-covered surfaces with boron layers. The recycling nature of 
carbon due to chemical erosion is supposed to explain the resilience of the core $\mathrm{C}$ concentration against the reduction of the primary sources [9], which is obvious from the pronounced reduction found in the inner divertor C deposition as shown in figure 1.
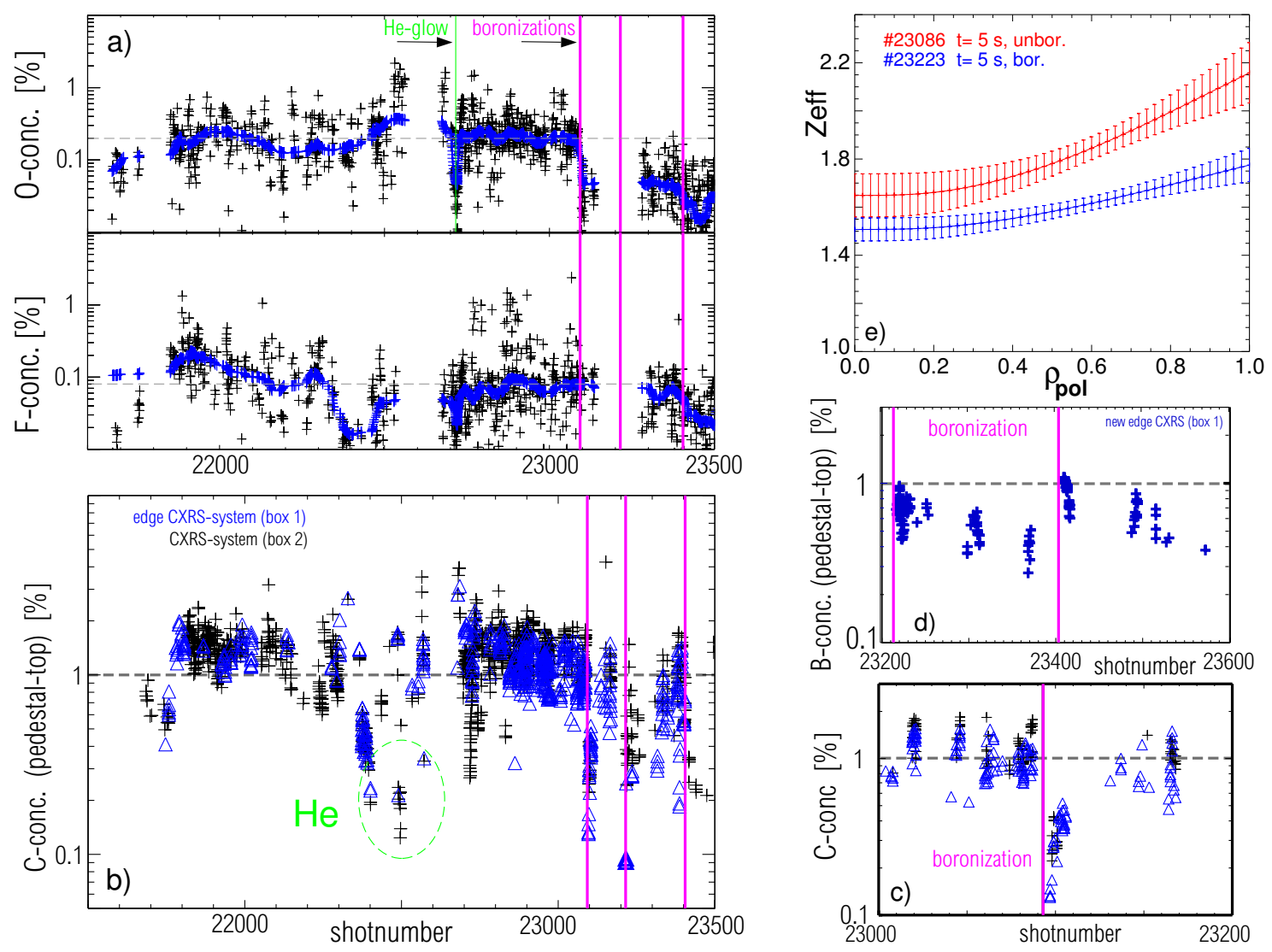

Figure 2. Development of impurity concentrations during the unboronized, full-W campaign and after boronization. a) $O$ and $F$ concentrations from X-ray lines measured by a Bragg crystal spectrometer $\left.\left(n_{e}>4.510^{19} \mathrm{~m}^{-3}\right) . b\right) C$ concentrations around the pedestal top measured by the core and edge CXRS systems $\left(n_{e}>410^{19} \mathrm{~m}^{-3}, P_{\text {heat }}>4.5 \mathrm{MW}, W_{M H D}>0.3 \mathrm{MJ}\right)$. c) expanded view of b) around the first boronization d) boron concentrations from CXRS after boronization e) $Z_{e f f}$ profiles from bremsstrahlung for two high power improved H-mode discharges before and after boronization.

The fluorine $(\mathrm{F})$ concentration shows little variation with boronisations. Our tentative explanation for the $\mathrm{F}$ source is thermal overload of teflon claddings of quartz fiber guides used for many spectroscopic diagnostics. This process is expected to correlate closely with the experimental parameters (heating power, fast ion losses), therefore little effect of boronisation is expected.

Many 'outliers' in the concentration plots can in fact be explained by special experimental conditions. E.g., experiments investigating the degradation of plasma confinement by elevated helium content [10] also showed reduced impurity concentrations. Radial profiles of $\mathrm{Z}_{\text {eff }}$ from bremsstrahlung are shown in figure $2 \mathrm{e}$ for representative high-power, improved $\mathrm{H}$-mode discharges before and after the first boronization. $\mathrm{Z}_{\text {eff }}$ is quite well reconciled by the spectroscopic measurements if the contribution of the individual impurities is summed up. A reduction of $Z_{e f f}$ after boronization was also reported from Alcator C-Mod [11], the additional contribution by boron being smaller than the effect of reduction of other intrinsic impurities. 


\section{Limitations of operational space}

The operational space of a full-W device is reduced in comparison to an all-carbon machine due to the possible occurence of central tungsten accumulation. Dedicated experiments were performed to map the operational space of the unboronized device, to understand the mechanisms leading to limitation of the operational space and to document its recovery after boronization.

\subsection{Central radiation limit due to $\mathrm{W}$ accumulation}

The most important limit for H-mode operation with tungsten PFCs is excessive central radiation due to tungsten accumulation close to the magnetic axis. $\mathrm{W}$ accumulation is caused by the neoclassical inward drift driven by the deuteron density gradient [12]. Figure 3 shows a typical example for tungsten accumulation in combination with central density peaking. The accumulation limit is a consequence of the tungsten source due to ELM-induced and inter-ELM sputtering by low-Z impurities [13], the impurity removal from the pedestal region by ELMs and the central power balance, which determines the anomalous transport level in the center. Important experimental actuators are ECRH heating (central power balance) and $\mathrm{D}_{2}$ gas puffing (ELM frequency, no pellet pacemaking was available in the 2007 / early 2008 campaigns). ICRH has currently a limited applicability for W acumulation avoidance since the strong W source induced by rectified sheath potentials [14] counteracts the beneficial effects of central heating.

Dedicated experiments with variations of the central ECRH heating power and gas flux resulted in a comprehensive experimental characterisation of the $\mathrm{W}$ accumulation limit. Figure 4 shows four unboronized discharges with different combinations of central ECRH and gas puffing at the edge of the stable operational domain. Two discharges with sufficient central ECRH and gas puff are stable, while the discharges with the lowest gas puff and the lowest ECRH power showed central density peaking in combination with $\mathrm{W}$ accumulation. A runaway situation is obtained when the tungsten radiation in the plasma center approaches a considerable fraction of the heating power flow. Under these conditions, the anomalous
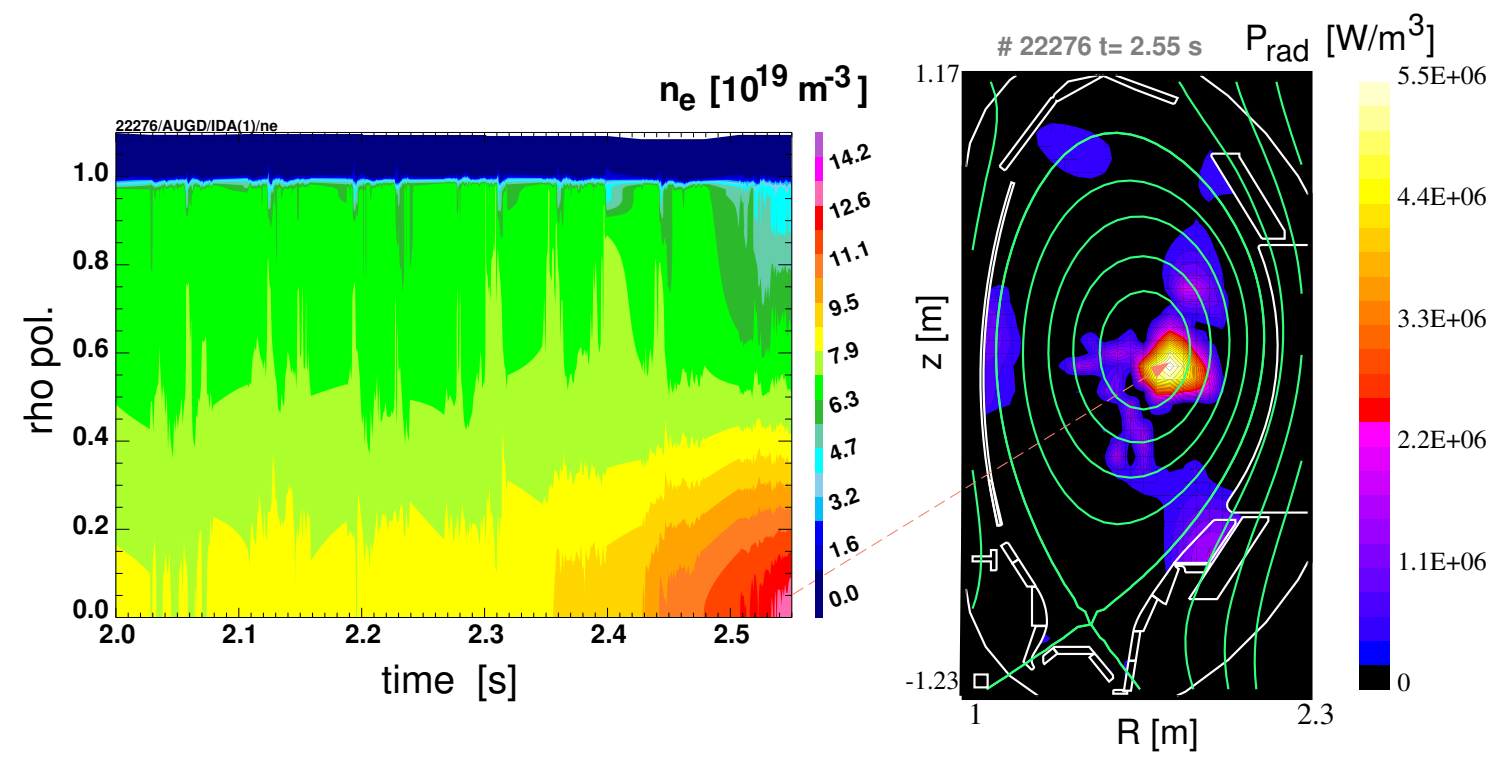

Figure 3. Density profile evolution and reconstructed radiated power distribution for a discharge with central density peaking and tungsten accumulation due to insufficient central heating and/or insufficient gas puffing, see figure 4.

transport is reduced, and the (deuteron) density profile peaks [15]. The peaking leads to an increased neoclassical inward 

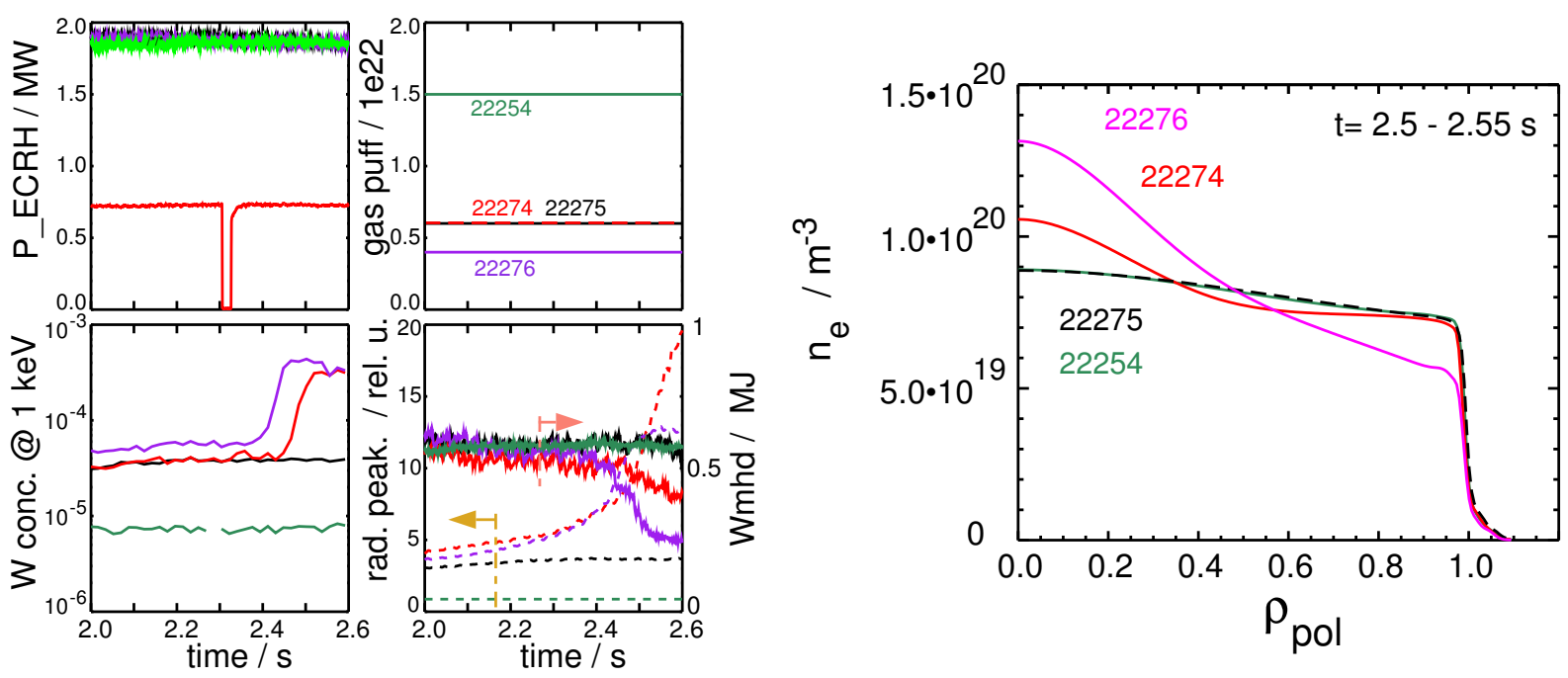

Figure 4. Time traces and density profiles of 4 discharges with different levels of central ECRH heating and gas puff. Cases with too low gas puff or ECRH power exhibit density peaking, central W accumulation and finally an H-L transition. $P_{N B I}=5 M W, I_{p}=1 M A$.

flux of tungsten, leading to increasing radiated power. Finally, the conducted power flow becomes too low to sustain the H-mode, and a H-L transition occurs. Usually, after an L-mode phase most of the tungsten is lost from the core and the discharge re-enters the H-mode. For low enough tungsten concentrations, stable central peaking of tungsten is also observed, which is possible as long as the central power balance is not disturbed too much. Scans of the ECRH deposition radius revealed that a very central deposition (inside $\rho_{p o l}=0.2$ ) is essential to prevent density peaking [16].

\subsection{Operational space of full-W ASDEX Upgrade with and without boronization}

To demonstrate the differences between non-boronized and boronized conditions, figure 5 compares two high performance discharges performed before and shortly after a boronization. The freshly boronized discharge showed stable behaviour without gas puffing, while under non-boronized conditions a deuterium gas puff of at least $3 \cdot 10^{21}$ atoms/s was required to avoid density peaking and $\mathrm{W}$ accumulation.

The reduced radiative losses after boronization, which are in line with the impurity composition behaviour shown in figure 2, lead to an increased divertor power load. Figure 6 shows the corresponding power loads obtained from IR thermography. The higher power load during boronized discharges with high heating power (above about $8 \mathrm{MW}$, depending on D gas puff rate) appeared to be above the limit for some outer divertor target tiles with VPS tungsten coating. The occurence of layer delamination for a few tiles required a vent for their exchange and strict divertor power limitations afterwards, see section 6.

Figure 7 shows the operational space of full-W AUG in terms of neutral gas flux and power flow to the divertor target, expressed by the difference of heating power and total radiated power. The upper limit of $\mathrm{P}_{\text {heat }}-\mathrm{P}_{\text {rad }}$ is a machine limit and corresponds to a time-averaged power density at the outer divertor of about $10 \mathrm{MW} / \mathrm{m}^{2}$. This limit increases slightly with the neutral gas level due to the broadening of the target power load profile with density / neutral flux [17]. The lower limit for $\mathrm{P}_{\text {heat }}-\mathrm{P}_{\text {rad }}$ can be the $\mathrm{H}-\mathrm{L}$ power threshold, but here the $\mathrm{W}$ accumulation limit is also important, since the low type-I ELM frequency at small separatrix power flows produces an experimental situation prone to $\mathrm{W}$ accumulation. At high values of the divertor neutral flux and low power, the type-III ELMy regime is located. Here, density peaking and $\mathrm{W}$ accumulation are observed also at high (type-III) ELM frequencies. However, due to the reduced confinement 
Article:
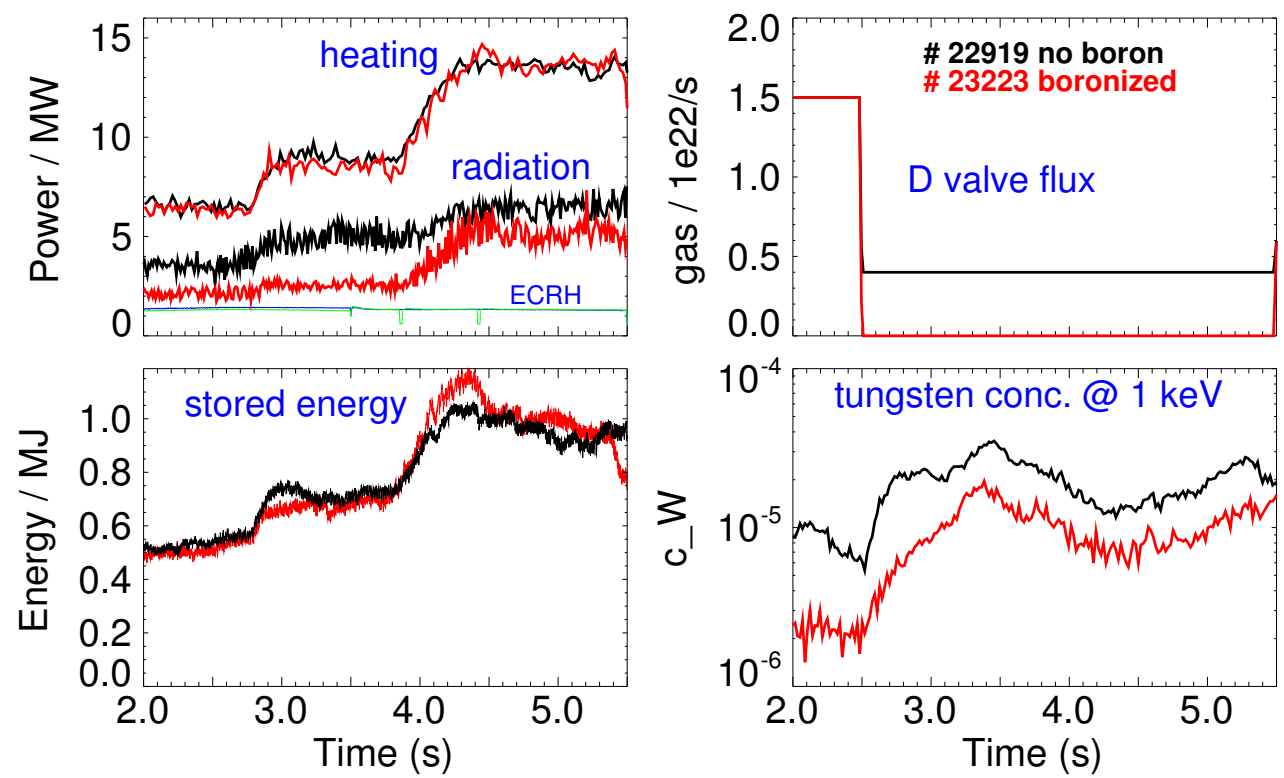

Figure 5. Comparison between high power improved H-mode discharges with identical heating schemes under non-boronized and boronized conditions. Shown are the heating power (almost identical) and total radiation time traces, the gas puff, stored energy and tungsten concentration. The H-factor $H_{98 y, 2}$ at $t=4.3 \mathrm{~s}$ is 1.1 for the unboronized and 1.3 for the boronized discharge without D-puff. For the latter discharge, confinement is slightly decreased by moderate neoclassical tearing mode activity. $I_{p}=1$ MA, $q_{95}=4.7$.

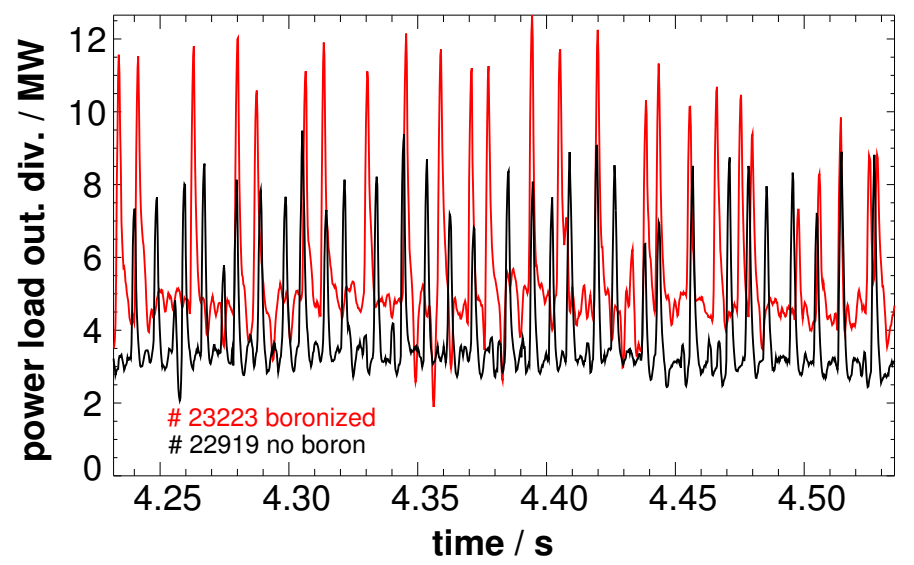

Figure 6. Total power load to the outer divertor from IR-thermography for the discharges of figure 5.

(typically $\mathrm{H}_{98 y_{2}}$ below 0.9 ), this regime is not of primary interest. Of particular importance are the discharges with W accumulation at higher values of $\mathrm{P}_{\text {heat }}-\mathrm{P}_{\text {rad }}$. With one exception, these discharges were run without boronization. The $\mathrm{W}$ accumulation is supposed to be caused by insufficient central heating power in view of the actual impurity content. In the high power operational domain, there is a wide window below the maximum power flux where stable operation is achieved and no influence of the boronization state is observed. More information about the operational space and relevant plasma parameters of the discharges shown in figure 7 are given in figure 8. For the predominantly type-I ELMy discharges without boronization, an ELM frequency above about $100 \mathrm{~Hz}$ is required for stable operation. Analogously, a corresponding high neutral density or gas puff rate is required. On the right figure 8, it is clearly shown that the central radiation strongly correlates with the tungsten concentration. Quantitative analysis shows that for the high radiation levels, tungsten radiation dominates the central radiative losses [19]. 


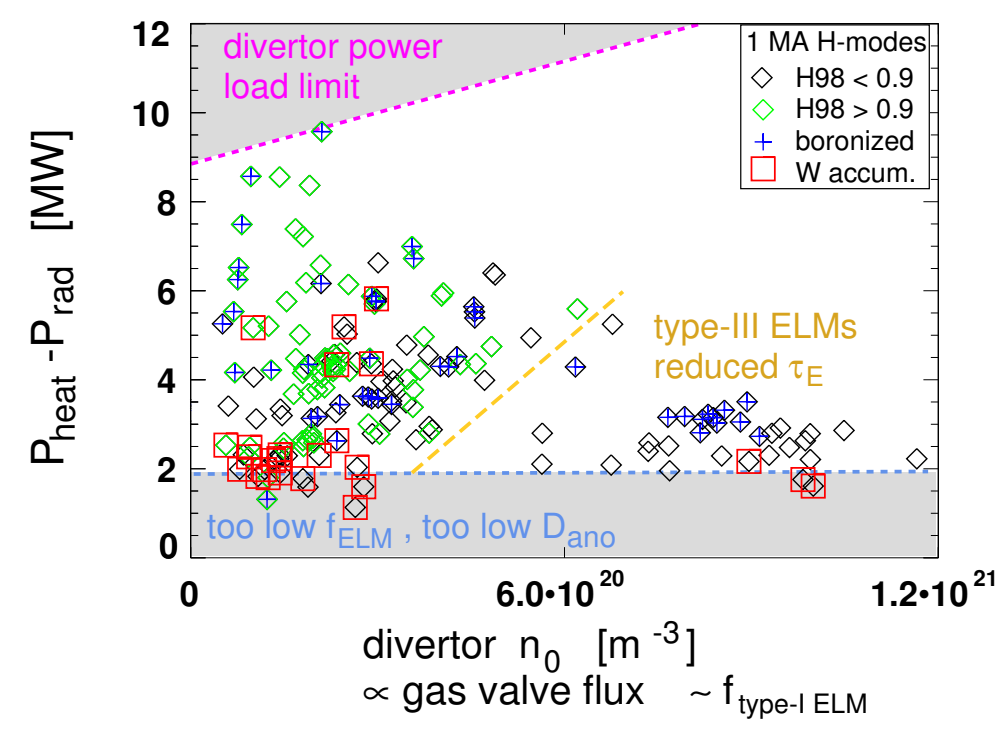

Figure 7. H-mode operational space in terms of divertor neutral flux and target heat load, $P_{\text {heat }}-P_{\text {rad. }}$ Data points represent either steady state flattop phases or phases leading to central W accumulation (red squares), averaging time is typically 0.5 s. The divertor neutral density, $n_{0, d i v}$, is nearly proportional to the total gas valve flux. Black diamonds denote discharges with $H$-factors $H_{98 y 2}$ below 0.9, which are typically type-III [18]. The limits indicated are just sketches guided by the data points. Plasma current $I_{p}=1$ MA.

In the stable part of the operational domain of AUG (see figure 7), no impact of the boronization state on energy confinement is observed. This is in contrast to results from the molybdenum covered Alcator C-Mod, where a clear reduction of the confinement with unboronized conditions was reported [20]. The confinement degradation in C-Mod has been attributed to high core molybdenum concentrations and corresponding radiative cooling. The more positive behaviour in AUG is at least partly explained by the availability of ECRH and high power NBI heating systems. Alcator C-Mod has to rely on ICRF for high power heating, which is prone to RF sheath induced sputtering of high- $Z$ wall material.
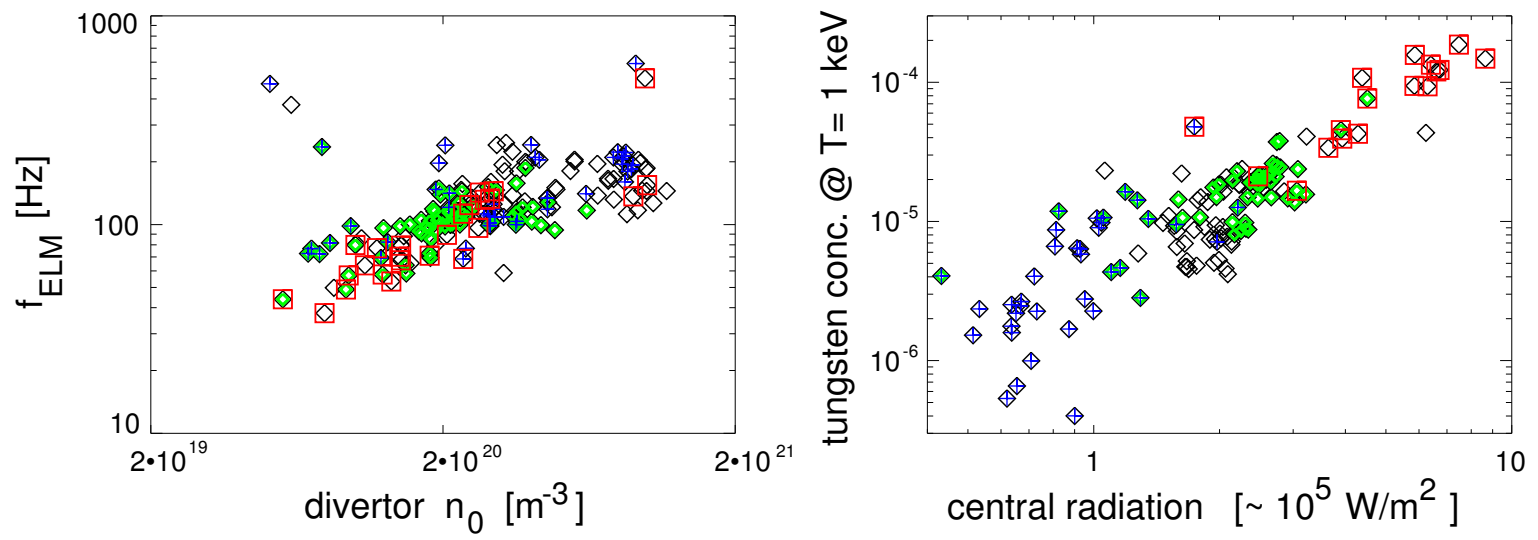

Figure 8. ELM frequency versus divertor neutral density (left) and tungsten concentration derived from the W quasicontinuum emitting around $T_{e}=1 \mathrm{keV}$ versus line integrated radiation from a central bolometer chord(right). same data points and symbols as in figure 7. Note that during $W$ accumulation, the quasicontinuum emission region broadens and shifts inward. 


\section{Tungsten source distribution and contribution to core $W$ content}

To improve operation of a full-W tokamak, and to overcome as far as possible the negative impact of strong W sources, knowledge about the spatial distribution of the sources and their relative impact on central impurity concentration is essential. Detailed spectroscopic analysis of the tungsten source distribution revealed considerable variations of source strengths and their impact on core W concentration [13]. The strongest W source is the outer divertor, but it has a small contribution to the core $\mathrm{W}$ content. Inner wall and outer limiters supply about similar W influxes, depending on the relative inner and outer wall gaps (see figure 9 for variation of distance to outer limiters). A significant fraction of the $\mathrm{W}$ source on all PFCs is caused by ELMs, with a higher relative contribution at lower inter-ELM edge temperatures. The core $\mathrm{W}$ content is usually dominated by the LFS limiter W source. The large penetration probability for W sputtered on the outer limiters poses a problem to ICRF operation in an all-W device. W sputtered by sheath rectified voltages caused predominantly by antenna box currents [21] [22] can lead to central $\mathrm{W}$ radiation comparable to the absorbed ICRF power for unboronized conditions. After the boronization, more favourable conditions with ICRF have been obtained, even after the boron coating has been eroded from the limiters. Since W sputtering with ICRH is also dominated by impurities, their lower concentration after boronization explains this observation.

Despite the significant contribution of ELMs to the sputtered W flux, increasing the ELM frequency always leads to a reduction of the $\mathrm{W}$ content due to the flushing of the impurities out of the pedestal region [23], [19]. This is demonstrated in figure 9. The line integrated SXR intensity shown for the shots with lowest and highest ELM frequency on the r.h.s of figure 9 shows clearly the pronounced impurity rise in between ELMs around the pedestal top. Although the stronger low-frequency ELMs produce a larger drop in the SXR emission, a step-wise rise of the emission level occurs. Comparing the rise in SXR emission at the pedestal top for the different ELM frequencies, a similar rise rate is observed after the ELM crash. This similar SXR rise rate in combination with a similar W source suggests that in fact the impurity transport through the edge transport barrier region is responsible for the rise in core W content with reduced ELM frequency. A possible screening of $\mathrm{W}$ in the SOL plasma due to the higher edge density caused by the higher gas puff appears to be less important in case of the main chamber source. The strong sensitivity of the core tungsten content on the ELM frequency is thought to be connected to the strong neoclassical inward pinch over the steep density gradient in the edge transport barrier region [ ?] [24]. Since the particle loss per ELM rises with reduced type-I ELM frequency [17], the total ELM induced particle loss flux changes only weakly with ELM frequency. Therefore, inter-ELM transport effects are thought to contribute to the almost inverse relation of ELM frequency and tungsten concentration for the conditions of figure 9. Tungsten ions accumulated at the pedestal top have more time to diffuse further into the core at reduced ELM frequency.

\section{Divertor temperature control for target power load reduction}

The reduced intrinsic radiation level after boronization of the machine resulted in peak target power loads in excess of the power limits of a few outer target tiles with thick VPS coating. As a consequence, control of the divertor power flux by impurity seeding had to be routinely used for high power discharges. To facilitate this, a previously developed feedback control procedure [25] using a (thermo-)electric current measurement in the outer divertor has been upgraded and implemented into standard AUG operation. The total inter-ELM charge flux at one target is interpreted to be thermoelectric and mainly driven by the electron temperature difference between outer and inner divertor plasma [26]. For standard toroidal field direction, there is net electron flux into the outer and net ion flux towards the inner target. The current loop is closed along the SOL plasma, the inner divertor and the conducting divertor structure [27]. Since the relevant quantity for divertor protection, the time-averaged peak power load at the outer divertor, scales roughly with the divertor temperature, 

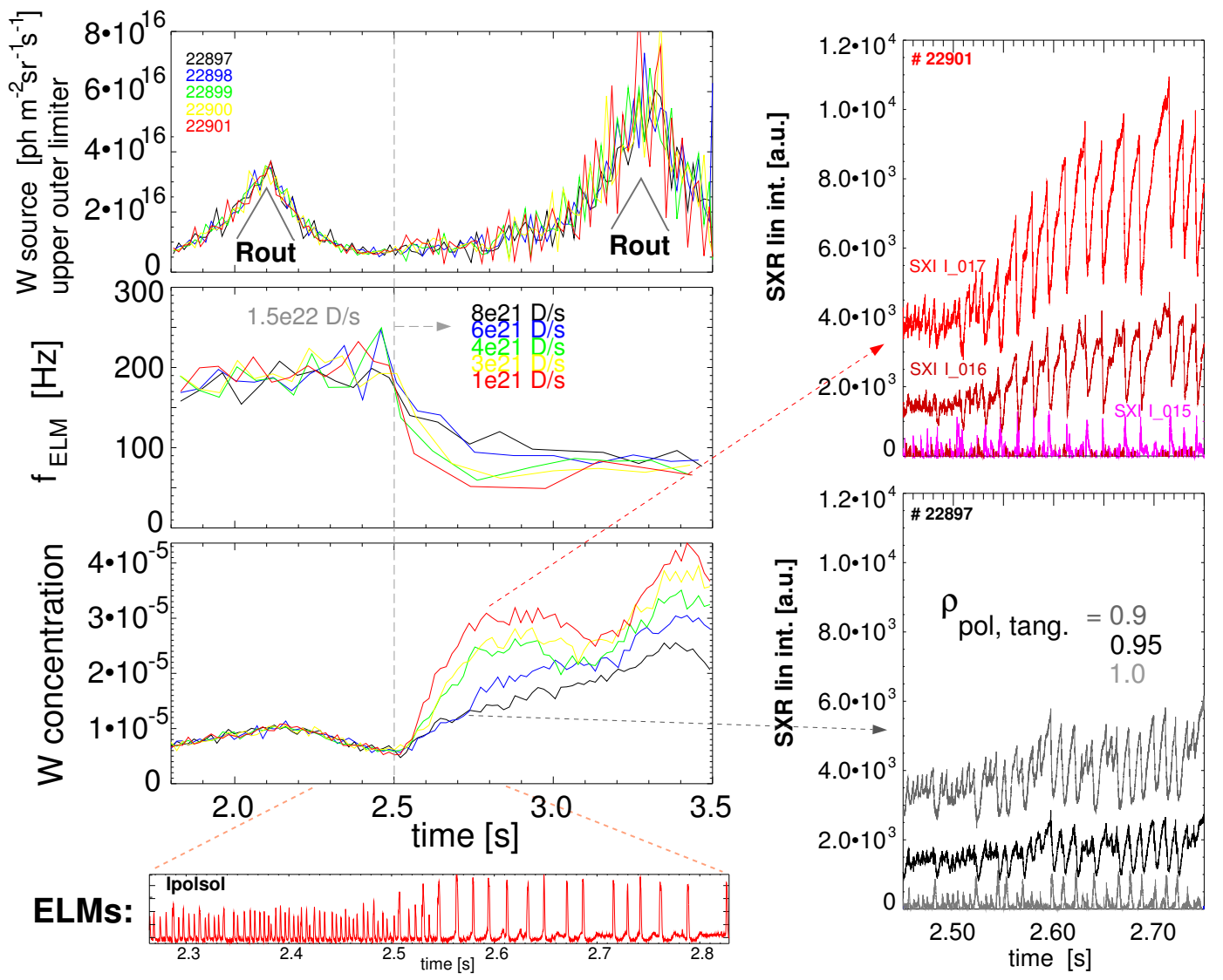

Figure 9. Variation of core W concentration @ $T_{e} \approx 1 \mathrm{keV}$ with ELM frequency for unboronized operation. $f_{E L M}$ has been varied by reducing the gas puff to different values. The tungsten source, as measured at the upper part of the outer limiter, remains constant over the change of $f_{E L M}$. An increase of the source is observed during an outward shift of the plasma column (Rout $\Lambda$ ) closer towards the limiters. $P_{\text {heat }}$ is $6.5 \mathrm{MW}$ before $t=2.8 \mathrm{~s}$ and $9 \mathrm{MW}$ afterwards, $P_{\text {rad }}$ rises from 3.5 to $4.5 \mathrm{MW}$ with the additional NBI source switched on a $t=2.8$ s. On the r.h.s., the line integrated SXR emission is shown for 3 edge channels with tangency radii of $\rho_{\text {pol }}=0.9,0.95$ and 1 for the discharges with the lowest (top) and highest (bottom) D fueling rate.

the easily available shunt measurement of the target current is a very appropriate diagnostic for feedback control. Real time processing of the target signal acquired at $100 \mathrm{kHz}$ with a cycle time of $1 \mathrm{~ms}$ using LabVIEW RT allowed to remove the effect of ELMS by a median filter. The feedback system appears to be very useful to control attached and partially detached divertor conditions, the latter regime being sufficient for the target load protection. For a fully detached divertor, the electric current in between ELMs vanishes completely, impeding further active control.

Nitrogen injection has been chosen for radiative cooling since $\mathrm{N}$ is more pronouced edge and divertor radiator compared to $\mathrm{Ne}$ and $\mathrm{Ar}$ [28]. The nitrogen gas is injected through 8 toroidally distributed nozzles through the divertor roof baffle towards the X-point. Figure 10 compares 2 otherwise identical discharges with and witout nitrogen seeding for target load control. The heating power was restricted to a total value below $10 \mathrm{MW}$ in order to allow for the unseeded pulse not to be stopped by the video target protection system. Analysis of target Langmuir probe data showed reduced $\mathrm{T}_{e}$ throughout the outer divertor with $\mathrm{N}$ seeding and partial detachment on the first $2 \mathrm{~cm}$ outside the outer strike point. The inner target is fully detached for both conditions. Preliminary analysis of bremsstrahlung measurements results in line-averaged $Z_{e f f}$ values of $1.5($ no $\mathrm{N}$ ) and 2 (with $\mathrm{N}$ ) between $\mathrm{t}=4$ and $4.5 \mathrm{~s}$ in the discharges shown in figure 10 . The confinement improvement observed is caused by increased temperature throughout the core plasma, while no significant changes are observed in the 

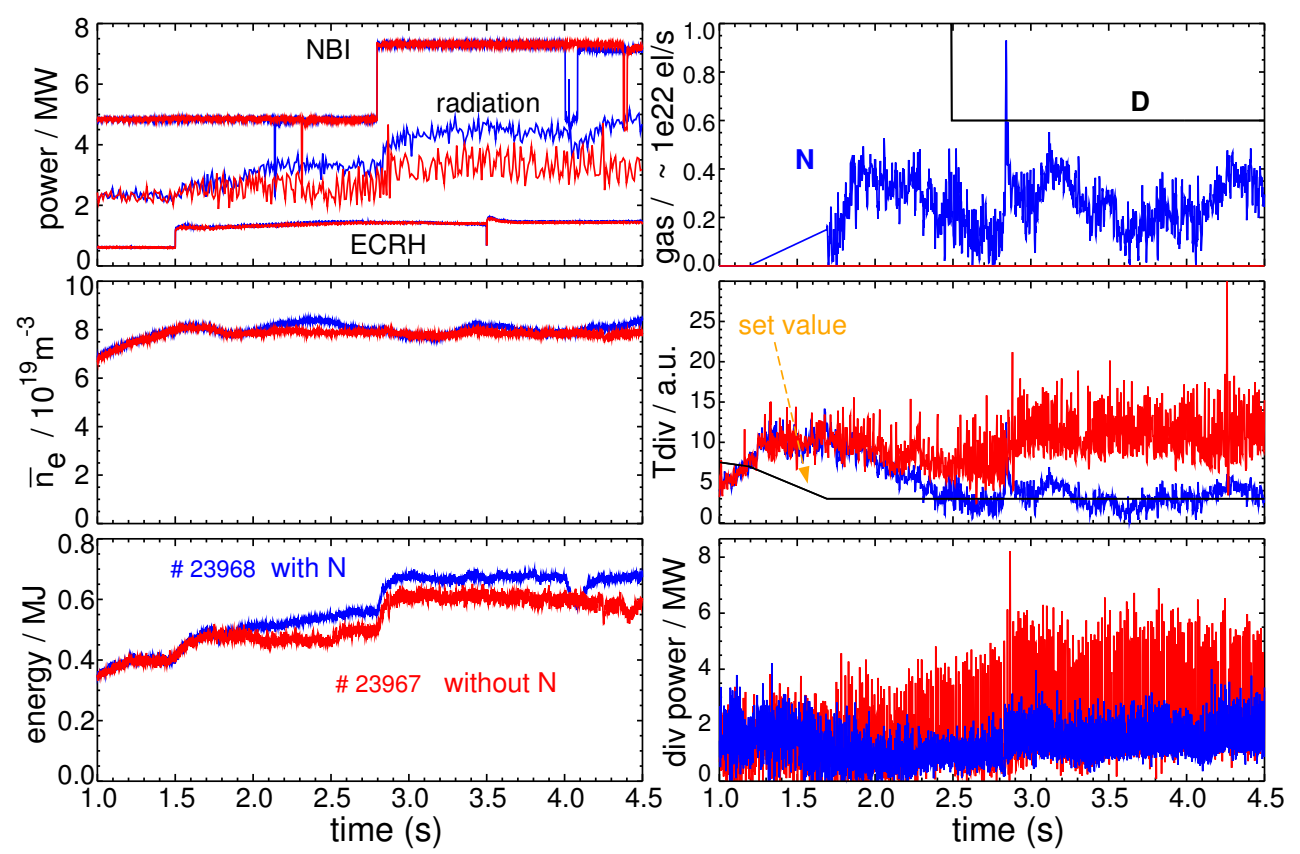

Figure 10. Comparison between two standard H-mode discharges with and without feedback-controlled nitrogen seeding. The (thermo-) electric current signal, Tdiv, approximately follows the total target power load measured by IR thermography. The ELM size is also reduced during nitrogen injection. $I_{p}=1 M A, q_{95}=4.6$.

core density profiles.

Figure 11 displays the radiation distribution of the discharges shown in figure 10 in comparison to a non-boronized discharge with the same D puff. The boronized discharge has lower main chamber radiation, as expected from the lower impurity content. With nitrogen seeding, the higher total radiation level of the non-boronized discharge is reproduced, albeit with a different spatial distribution: Nitrogen enhances just the divertor and lower scrape-off layer radiation.

The ELMs with nitrogen seeding appear much smaller than for similar radiation levels under intrinsic, unboronized conditions. Figure 12 shows coherent ELM averaged [17] measurements of the time evolution of target power load and the total electric current in the outer divertor for the discharges of figure 10. The current measured simultaneously at the inner target (not shown) has the opposite sign but almost identical value, as expected for the single-null configuration. ELM size variations show up quite similar in the electic current and IR power load measurements. The electric current during the ELM is supposed to have a strong contribution of ions which hit the inner divertor and carry a substantial fraction of the ELM energy [17]. Assuming that the relative contributions to the ELM loss (ions versus electrons, high-field side versus low-field side) do not change significantly with ELM size for the present conditions, the similar relative variations of power load and current can be understood.

The nitrogen seeding has two counteracting effects on the tungsten source. The additional sputtering of W by the impact of $\mathrm{N}$ ions increases the $\mathrm{W}$ source, while the effect of edge cooling reduces the $\mathrm{W}$ source due to the lower energy of the impacting ions. For the discharges of figure 10, these effects approximately cancel. The total divertor neutral $\mathrm{W}$ source remains the same $\left(210^{19} \mathrm{~W} / \mathrm{s}\right.$ at $\left.\mathrm{t}=3.9 \mathrm{~s}\right)$, while the limiter source is slightly enhanced for the discharge with $\mathrm{N}$ seeding $\left(2.410^{18} \mathrm{~W} / \mathrm{s}\right.$ vs. $\left.1.810^{18} \mathrm{~W} / \mathrm{s}\right)$. The tungsten concentration profile in the central plasma is flat around $\mathrm{t}=4 \mathrm{~s}$ for both discharges with values $c_{W}=1.510^{-5}$. The slightly higher $\mathrm{W}$ limiter source with $\mathrm{N}$ seeding is assumed to be compensated by the slightly higher ELM W flushing frequency.

The wall storage behaviour of nitrogen in the boronized full-W device turned out not to limit experimental operation 

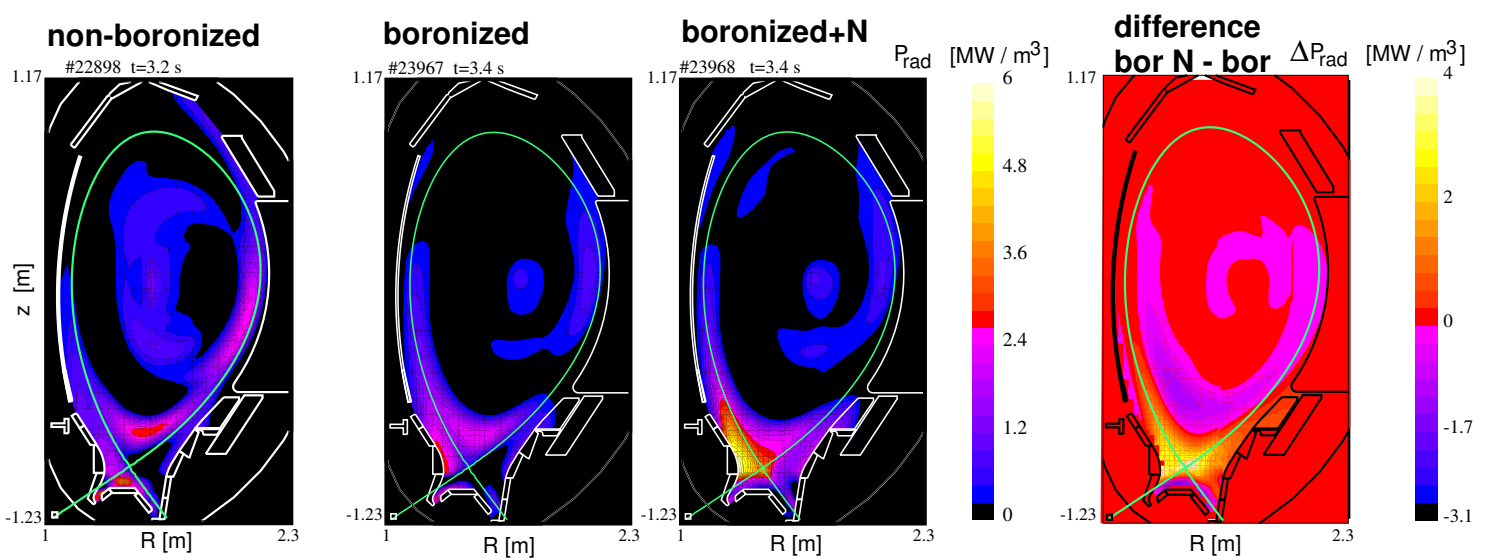

Figure 11. Comparison of the radiation distribution from bolometry of 3 discharges with identical heating power and D fuelling for non-boronized and boronized conditions with and without nitrogen seeding. For the latter cases, also the difference in radiation pattern is shown. The total radiated power are 4.5 MW for the non-boronized and the $\mathrm{N}$-seeded discharge and $3.4 \mathrm{MW}$ for the boronized discharge.
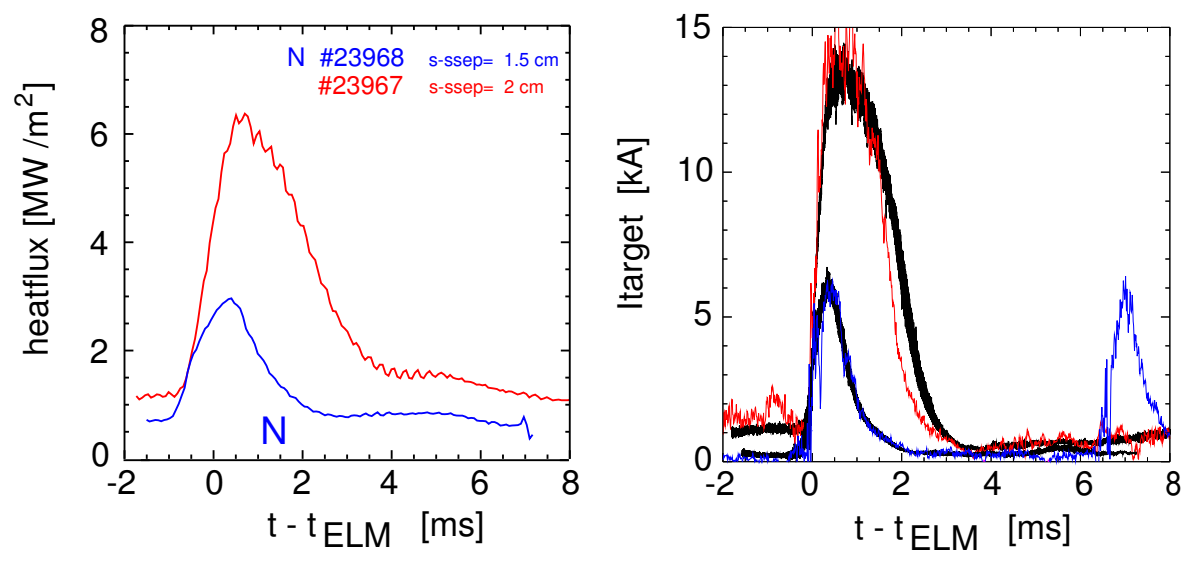

Figure 12. Coherent ELM averaged power flux density (left) at the position of maximum power flux close to the strike point and electic current measured by shunts in the outer divertor (right) for the discharges of figure 10. On the right graph, black lines represent the coherent average wrt the ELM start time and the colored lines time traces of 2 typical individual ELMs. Power flux and electric current exhibit quite similar signatures. The IR diagnostic has a slower time resolution (260 $\mu$ s) compared to the shunt measurement (10 $\mu$ s).

even in long series of discharges with $\mathrm{N}$ seeding without any inter-discharge glow conditioning. Neither excursive $\mathrm{N}$ wall release nor plasma breakdown or $\mathrm{I}_{p}$ buildup problems occured. However, discharges without active seeding done after seeded plasmas showed nitrogen levels of about $50 \%$ in the subsequent or about $30 \%$ in the third pulse.

\section{Conclusions}

Operation of a full-metal device without boronization is the only way to study fuel retention under conditions of very small co-deposition. In fact, the codeposition of D with low-Z impurities in AUG could be reduced so far that deep trapping of hydrogen in the bulk material became a relevant long-term storage mechanism, with retention rates well below a percent of the gas input.

Since sputtering by low- and medium-Z impurities dominates the erosion of tungsten, the H-mode operational window in 
terms of mimimum required values of gas puffing and central heating is somewhat reduced comparing un-boronized with boronized operation. The operational limit is set by the occurrence of central tungsten accumulation and central radiative losses comparable to the local heating. The limit is understood as a consequence of the tungsten source, predominantly at the low field side limiters, the frequency and size of ELMs flushing the tungsten ions out of the pedestal region and the central anomalous transport level which counteracts the neoclassical inward drifts. A higher impurity level may increase or decrease the tungsten source, depending on the counteracting effects of increased impurity fluxes and reduced edge temperatures. However, core impurity radiation always tends to reduce the ELM frequency due to the reduction of the power flux through the separatrix. Comparing boronized with unboronized operation, the effect of the respective impurity compositions is that with boronization, a reduced D puff or reduced central heating power is tolerated. In contrast to results from Alcator C-Mod, no confinement degradadation is observed in unboronized AUG operation.

Due to the decreased impurity content after boronization, the divertor power load becomes the limiting quantity for operation and seed impurities have to be added to the plasma for radiative power exhaust. While seed impurities produce competing effects with regard to the tungsten source (high impurity flux to the wall, but reduced edge temperature and impact energy), the reduction of ELM frequency caused by the additional core radiation (in case of $\mathrm{Ne}$ and Ar injection) leads to less favourable impurity transport. With reduced $\mathrm{f}_{E L M}$, the strong neoclassical inward drift for tungsten in the edge transport barrier region is not compensated by ELM flushing out of the pedestal region. Therefore, Ne and Ar seeding is only possible in combination with ELM pacemaking, e.g. by pellet injection [29].

An exception with impurity seeding is nitrogen. Nitrogen radiates predominantly in the SOL, private flux and divertor region and therefore is much more tolerated by the plasma in terms of absence of tungsten accumulation. There is also a slight rise of $\mathrm{f}_{E L M}$ in combination with reduced ELM size during strong nitrogen puffing, an observation which has not been understood so far. With nitrogen seeding, at most slightly enhanced central heating or deuterium puffing are required in comparison to unseeded/unboronized conditions to obtain stable discharges with low central tungsten content. As a result, target power load reduction with nitrogen allows to increase the core heating power considerably. So far, $15 \mathrm{MW}$ could be injected into AUG while keeping the time-averaged peak power load in the outer divertor as low as $5 \mathrm{MW} / \mathrm{m}^{2}$, the heating power currently being limited only by the available flywheel generator power.

In order to investigate the impact of boronization in very high power discharges, another unboronized operation of AUG is planned when flywheel generator EZ4 is available again in 2009. Future studies will cover the effect of resonant magnetic perturbations used for ELM control starting end 2010. A high central heating power turned out to be very beneficial for a high-Z machine. Therefore, the ECRH system will be further enhanced, and modifications of the ICRF antennas are under development [30], [31], which are expected to significantly reduce the tungsten sputtering rate at the end of field lines crossing the antenna boxes.

\section{Acknowledgement}

This work, supported by the European Communities under the contract of Association between EURATOM/IPP, was carried out within the framework of the EFDA Task Force on Plasma Wall Interactions. The views and opinions expressed herein do not necessarily reflect those of the European Commission.

\section{References}

[1] NEU, R. et al., Plasma Phys. Controlled Fusion 49 (2007) B59. 
[2] NEU, R. et al., J. Nucl. Mater. 367-370 (2007) 1497.

[3] BOBKOV, V. V. et al., J. Nucl. Mater. 363-365 (2007) 122.

[4] MAYER, M. et al., Nucl. Fusion 47 (2007) 1607.

[5] MAYER, M. et al., Carbon balance and deuterium inventory from a carbon dominated to a full tungsten ASDEX Upgrade, PSI 2008 conference, subm. to Journ. Nucl. Mat.

[6] ROHDE, V. et al., Dynamic and static deuterium inventory in asdex upgrade with tungsten first wall, in in Fusion Energy 2008 (Proc. 22nd Int. Conf. Geneva, 2008) (Vienna: IAEA) CD-ROM file EX/P4-3 and http://wwwnaweb.iaea.org/napc/physics/FEC/FEC2008/html/index.htm

[7] OGORODNIKOVA, O. et al., J. Nucl. Mater. 313-316 (2003) 469.

[8] ROTH, J. et al., Recent analysis of key plasma wall interaction issues for ITER, in PSI 2008 conference, accepted for publication in J. Nucl. Mat., 2008.

[9] KALLENBACH, A. et al., J. Nucl. Mater. 363-365 (2007) 60.

[10] NEU, R. et al., Influence of the ${ }^{4} \mathrm{He}$ concentration on H-mode confinement and transport in ASDEX Upgrade, in Europhysics Conference Abstracts (CD-ROM, Proc. of the 35rd EPS Conference on Plasma Physics, Hersonissos, Crete, 2008), volume 32F, pages P-4.039, Geneva, 2008, EPS.

[11] LIPSCHULTZ, B. et al., J. Nucl. Mater. 363-365 (2007) 1110.

[12] DUX, R. et al., Plasma Phys. Controlled Fusion 45 (2003) 1815.

[13] DUX, R. et al., Plasma-wall interaction and plasma behaviour in the non-boronised all tungsten ASDEX Upgrade, PSI 2008 conference, subm. to Journ. Nucl. Mat.

[14] DUX, R. et al., J. Nucl. Mater. 363-365 (2007) 112.

[15] NEU, R. et al., J. Nucl. Mater. 313-316 (2003) 116.

[16] GRUBER, O. et al., Compatibility of iter sceanrios with full tungsten wall in ASDEX Upgrade, in in Fusion Energy 2008 (Proc. 22nd Int. Conf. Geneva, 2008) (Vienna: IAEA) CD-ROM file EX/1-5 and http://wwwnaweb.iaea.org/napc/physics/FEC/FEC2008/html/index.htm

[17] KALLENBACH, A. et al., Nucl. Fusion 48 (2008) 085008.

[18] RYTER, F. et al., Journal of Physics: Conference Series 123 (2008) 012035.

[19] KALLENBACH, A. et al., Plasma Phys. Controlled Fusion 47 (2005) B207.

[20] LIPSCHULTZ, B. et al., Nucl. Fusion 47 (2007) 1189.

[21] COLAS, L. et al., Plasma Phys. Controlled Fusion 49 (2007) B35.

[22] BOBKOV, V. et al., Operation of icrf antennas in a full tungsten environment in ASDEX Upgrade, PSI 2008 conference, subm. to Journ. Nucl. Mat.

[23] WADE, M. R. et al., Phys. Rev. Lett. 94 (2005) 225001.

[24] PÜTTERICH, T. et al., Fast cxrs-measurements in the edge transport barrier of ASDEX Upgrade, in Europhysics Conference Abstracts (CD-ROM, Proc. of the 35rd EPS Conference on Plasma Physics, Hersonissos, Crete, 2008), volume 32F, pages $\mathrm{P}-$ 2.083, Geneva, 2008, EPS.

[25] KALLENBACH, A. et al., J. Nucl. Mater. 337-339 (2005) 732.

[26] STAEBLER, G. M. and HINTON, F. L., Nucl. Fusion 29 (1989) 1820.

[27] KALLENBACH, A. et al., J. Nucl. Mater. 290-293 (2001) 639.

[28] KALLENBACH, A. et al., Plasma Phys. Controlled Fusion 38 (1996) 2097.

[29] LANG, P. T. et al., Nucl. Fusion 45 (2005) 502.

[30] BOBKOV, V. et al., Calculations of near-fields of ICRF antenna for ASDEX Upgrade, in Europhysics Conference Abstracts (CDROM, Proc. of the 35rd EPS Conference on Plasma Physics, Hersonissos, Crete, 2008), volume 32F, pages P-5.005, Geneva, 2008, EPS.

[31] BOBKOV, V. et al., Assessment of compatibility of ICRF antenna operation with full W-wall in ASDEX Upgrade, in in Fusion Energy 2008 (Proc. 22nd Int. Conf. Geneva, 2008) (Vienna: IAEA) CD-ROM file EX/P6-31 and http://wwwnaweb.iaea.org/napc/physics/FEC/FEC2008/html/index.htm 